\section{The magical number fourteen: Making a very great deal of non-sense}

\author{
A. W. MacRAE \\ The University of Birmingham, Birmingham, England
}

Norwich (1981) sets out to develop, by a mathematical argument extending over 14 pages, a quantitative theory relating performance on various sensory tasks to the adaptation rates of sensory neurons. However, the paper has several important flaws which entirely invalidate its conclusions.

\section{The "Psychophysical Adaptation Curve"}

A key idea in the paper is what Norwich calls a "psychophysical adaptation curve." It represents the decline in stimulus equivocation (corresponding to the increase in transmitted information) as the duration of application of a stimulus increases. From where does Norwich obtain this curve? He imagines a series of absolute judgment experiments, following the paradigm of Garner and Hake (1951) and with different durations of exposure of the stimuli in each experiment. He concludes that "if we were to draw a graph of $\mathrm{H}_{\mathrm{s}}$ against stimulus duration, we should probably obtain something like the graph shown in Figure 1" (p. 410). We might, of course, but there could hardly be a more tenuous basis for a theoretical development.

\section{Psychophysical and Physiological Adaptation}

The implication that Norwich draws from the shape of this imagined curve is quite remarkable. His Figure 2 shows a schematic diagram of the decline with time in the rate of firing of a sensory neuron while the receptor receives a constant, uniform stimulus. The inference that follows had better be expressed in Norwich's own words to avoid any suspicion that he is being misrepresented:

The neuron will fire initially at maximum rate, progressively slowing its firing rate until it reaches an equilibrium level. The graph of firing rate against time describes the physiological adaptation curve of this sensory unit (Figure 2). The physiological adaptation curve has the same general shape as the psychophysical adaptation curve shown in Figure 1. It therefore seems reasonable to as-

This paper was completed while the author was a visiting professor at the University of Michigan, Ann Arbor. Thanks are tendered to the Department of Psychology at Ann Arbor, and especially to Clyde Coombs, for their hospitality and stimulus, and to the Human Factors Program of the Scientific Affairs Division of NATO who provided financial support for the visit. The author's permanent mailing address is Department of Psychology, University of Birmingham, P.O. Box 363, Birmingham, B15 2TT, England. sociate the firing rate of the neuron, $F$, with the entropy or stimulus equivocation, $\mathbf{H}_{\mathrm{s}}$. We write, then, by hypothesis (Norwich, 1977),

$$
\mathrm{F}=\mathrm{kH}_{\mathrm{s}},
$$

where $k$ is a constant that is greater than zero (p. 411).

In other words, two graphs have "the same general shape" and therefore they may be taken to represent identical functions. Norwich's Equation 6 assumes that the ratio of the heights of the two curves is constant at all times. Apart from the scale factor, $k$, the initial heights $\left(H_{s} \max\right.$ and $\left.F_{\max }\right)$, the asymptotes $\left(H_{s} \min\right.$ and $\left.F_{\min }\right)$ and the rates of change in $H_{s}$ and in $\mathrm{F}$ are assumed to be identical.

Now a graph showing the decay of a radioactive substance also has the same general shape as these curves, although the time scale might be measured in microseconds, or in centuries, rather than in seconds. But then, Norwich has offered no evidence that the time scales of his Figures 1 and 2 are in any way similar. Nor does he appear to think that time scales are important in this context. The unidentified time units in one set of neuronal adaptation data that he quotes run from .50 to 14.00 . In the other, they run from .025 to 1.900 .

Even if it seems a little less plausible to relate the change in identifiability of stimuli to a decline in radioactivity, it should be noted that the form of the argument would be the same as for Norwich's proposal. Neither is logically valid.

\section{The "Entropy Function for a Sensory Receptor"}

In the next section of the paper, Norwich attempts to find "the mathematical form of the entropy function, $\mathbf{H}_{\mathrm{s}}$, for a sensory receptor." The answer he comes up with is clearly incorrect.

At the end of a long chain of mathematical argument, he concludes that $F$, the firing rate of a sensory neuron, is given by the function:

$$
F=1 / 2 k \log _{e}\left(1+\frac{B}{\tau}\right) \frac{1}{\log _{e} 2},
$$

where $\mathbf{k}$ is the constant we met before, $B$ is another constant depending on the particular situation, and $\tau$ is a measure of time.

This function approaches infinity as $\tau$ approaches zero, and approaches zero as $\tau$ approaches infinity. Neuronal firing does not behave like that, as Norwich admits.

Of course, no real data ever conform exactly to a theoretical function, and it is quite possible for a function to give useful results if it provides even an 
approximate model for the important features of the data it represents. But what Norwich turns out to be interested in estimating is the ratio of the maximum to the minimum firing rate-the very quantities for which his Equation 15 cannot possibly be correct, however well it may fit in some places. ${ }^{.}$

He uses a curve-fitting technique to find values for $\mathrm{k}$ and $\mathrm{B}$ in Equation 15 to give best fits to some sets of data on neuronal adaptation in various species. On each fitted curve, he then selects two points that seem to him appropriate and characterizes them as the "maximum" and "minimum" of the curve. He gives no criteria for his selection, except to note that the values chosen lie "slightly" outside the measured ranges of frequency. In other words, they were determined principally by the range of time over which the original experimenters chose to make their measurements, with an intuitively based ad hoc adjustment by Norwich. Choosing different points on the curve could, of course, produce radically different estimates of the ratio between "maximum" and "minimum."

He makes no comment on the indeterminate nature of the maximum of the function, but he does make a special note that "because of the asymptotic approach of the adaptation curve to its lowest, or plateau, level, it is not necessary to apply a stimulus for an infinite period of time in order to make a reasonable estimate of the plateau level. A stimulus of finite duration will very soon produce a close estimate of $I_{t \max }$ " (p. 414).

This note seems to imply that he does not realize that the "plateau level" predicted by his function is known a priori. But on page 418 he devotes a paragraph to arguing that, although Equation 15 predicts that $F$ will approach zero for large values of $\tau$, the prediction does not conform to reality and there is a limitation on the size of $\tau$ that can enter Equation 15. But on page 418 he also speaks of a neuron "transmitting at a rate above its noise level," and says that for long periods of observation of a steady stimulus "the impulse transmission rate, F, approaches zero (above its spontaneous transmission rate)."

Thus, Norwich offers three conflicting interpretations of the relationship between firing rate and time: Equation 15, Equation 15 with a limit on $\tau$ (or a nonlinear function relating $\tau$ to chronological time), and Equation 15 with an added (baseline) constant. The first of these will not do because it does not fit the facts. The second and third could adequately describe the minimum of the curve (though not the maximum), but Norwich does not use them in interpreting the data he quotes. Nor do they follow from his argument. Equation 15 is not supposed to be approximate, because Norwich deduces it with every appearance of mathematical rigor. How can the result be wrong?

\section{Signal, Noise, and Information}

More than one error contributes to the nonsensical form of Equation 15, but the most fundamental errors relate to Norwich's misuse of the concepts of "signal" and "noise." He defines "the entropy or uncertainty about the mean, at the receptor" thus:

$$
\begin{aligned}
\mathrm{H}_{\mathrm{s}}= & -\int \mathrm{p}_{\mathrm{SN}}(\mathrm{I}) \log \mathrm{p}_{\mathrm{SN}}(\mathrm{I}) \mathrm{dI} \\
& +\int \mathrm{p}_{\mathrm{N}}(\mathrm{I}) \log \mathrm{p}_{\mathrm{N}}(\mathrm{I}) \mathrm{dI}
\end{aligned}
$$

where $p_{S N}(I)$ and $p_{N}(I)$ are the probability density functions of "signal plus noise" and of "noise," respectively.

Equation 8 would be appropriate if the terms " $\mathrm{H}_{\mathrm{s}}$," "signal," and "noise" had their usual meanings, but Norwich does not use them in the usual way.

The symbol " $\mathrm{H}_{\mathrm{s}}$ " usually denotes the information content of a varying signal, where variation in the signal is the object of prime interest. Information is transmitted when changes in the signal are responded to, and there is more information in a signal when there is greater signal variance. Informally, a larger number of different signal states can be identified reliably when the signal varies more widely.

In the situation supposedly described by Equation 8 , the quantity of prime interest is the mean value of a steady signal which, nevertheless, consists of a spectrum of different values. " $H_{s}$ " refers to uncertainty about the location of the mean. That uncertainty is greater when the spectrum is distributed about the mean with greater variance, so there is a close, though complementary, analogy between the two situations. But the situations differ radically in respect of the effects of noise.

In the standard situation, noise variance is intrinsically confusable with signal variance, so the presence of noise reduces the effective information in the signal and $\mathrm{H}_{\mathrm{s}}$ becomes smaller. Informally, fewer different levels of signal can be discriminated reliably when the signal is perturbed by noise. But in Norwich's situation, the presence of noise adds to the uncertainty of the signal mean and so $\mathrm{H}_{\mathrm{s}}$ becomes larger. Increasing the signal variance will have the same kind of effect as increasing the variance of the additional noise source that Norwich posits. Both will increase uncertainty.

Thus, Equation 8 is totally inappropriate, given the way that Norwich defines and uses his terms. Consequently, all the conclusions that flow from it, that is, all later parts of the paper, are unfounded.

Nor is that all. When Norwich derives Equation 10 from Equation 8, he fails to take account of the effect on the noise variance of taking multiple samples. Although that error may be somewhat less funda- 
mental than the other, it is equally devastating to the argument. If Equation 8 were, in fact, valid, then correcting the second error would remove the influence of sample size (and hence of time), on which the rest of the paper is predicated.

Finally, it may just be worth noting that Equation 8 is described as a definition of $\mathrm{H}_{\mathrm{s}}$. It is quite different from, and not necessarily consistent with, the definition for the same symbol given on page 410 , where $\mathbf{H}_{\mathrm{s}}$ was defined as the stimulus equivocation in a Garner-Hake experiment.

Thus, in this fundamental section of the paper there are several interlocking errors and confusions.

\section{Conclusion}

It does not seem profitable to continue with any detailed analysis of the paper beyond this point. Although there are many errors, it is not always possible to say which of them are crucial, because, as I have shown, the paper contains so many internal contradictions that it is often impossible to focus on a single interpretation. Thus, my contention is not that Norwich's paper as a whole is mistaken, but that it is nonsensical, having no clear meaning.

But I must mention one other, relatively trivial, error, since it relates to a paper of mine and silence might be taken to imply assent. The purpose of the paper by MacRae (1970) was not, as Norwich suggests, to show that the method used to calculate transmitted information carries a bias. That point had been made previously by others more competent than myself. The point of my paper was that the inter- action of that bias with the design of most absolute judgment experiments had generated purely artifactual asymptotes in the relationship between transmission and stimulus information. I concluded that there was therefore no evidence that performance in these tasks was limited by an information channel of fixed capacity. Of course, that conclusion might not be congenial to Norwich, since it may be thought to remove some of the magic from the number seven.

\section{REFERENCES}

Garner, W. R., \& HAKe, H. W. The amount of information in absolute judgments. Psychological Review, 1951, 58, 445-459.

MAcRAe, A. W. Channel capacity in absolute judgment tasks: An artifact of information bias? Psychological Bulletin, 1970, 73, 112-121.

NoRwich, K. H. On the information received by sensory receptors. Bulletin of Mathematical Biology, 1977, 39, 453-461 (as cited by Norwich, 1981).

Norwich, K. H. The magical number seven: Making a "bit" of "sense." Perception \& Psychophysics, 1981, 29, 409-422.

\section{NOTE}

1. In another paper in this issue (pp. 000-000), Norwich correctly points out that he made use of differences, and not ratios, between $F_{\max }$ and $F_{\min }$. The word "difference" should be substituted for the word "ratio" in this paragraph and the following one. None of this paper's conclusions are affected by the change. (Note added in proof.)

(Manuscript received November 19, 1981; revision accepted for publication February 12, 1982.) 